\title{
Editorial
}

\section{New Methods and Questions in}

\section{Metamemory Research}

\author{
Monika Undorf ${ }^{1}$ and Vered Halamish ${ }^{2}$ \\ 1 Department of Psychology, School of Social Sciences, University of Mannheim, Germany \\ ${ }^{2}$ School of Education, Bar-Ilan University, Ramat-Gan, Israel
}

Am I ready for the exam on Monday? Will I be ready for the exam if I study all weekend? Did I do well on the exam I've just submitted? Should I program my smartphone to remind me to buy milk? Have I remembered all the things I meant to buy, or should I check my shopping list? These examples illustrate that metamemory is ubiquitous in everyday life. Metamemory refers to people's knowledge about learning and memory processes in general and to the assessment (monitoring) and regulation (control) of these processes as they occur. Ever since Flavell (1971) coined the term metamemory and Nelson and Narens (1990) introduced a seminal theoretical framework to guide its investigation, metamemory has elicited a wealth of research. This research has been done by researchers from various disciplines including cognitive psychology, developmental psychology, educational psychology, and neuroscience, among others. The collection of papers in this topical issue of Zeitschrift für Psychologie provides an overview of methods and research questions that represent important new directions for metamemory research.

\section{New Methods}

Three articles in this topical issue propose new methods for the study of metamemory. In a review paper, Kelley et al. (2020) present computational neuroimaging approaches that can provide new insights into the processes, or algorithms, underlying metamemory. They further discuss how such new approaches could be applied to unresolved questions in the behavioral metamemory literature, thereby bridging experimental and neurocognitive metamemory research. Dobbins and Kantner (2020) promote analyzing the natural language people use to describe their experiences during remembering with statistical learning algorithms. This new "verbal reports as data" approach has the potential to shed light on the basis of a wide range of metamemory judgments without forcing people to use categorical ratings or experimenter-defined response options. Kälin and Roebers (2020) suggest that time-based measures can reveal aspects of children's memory monitoring that are not evident in children's explicit metamemory judgments. Consistent with this suggestion, they showed that retrospective confidence judgments were unrelated to executive functions, whereas time-based measures of monitoring were positively related with executive functions.

\section{Emerging Research Questions}

The other six articles in this topical issue expand our knowledge about metamemory by addressing current and emerging research questions.

One of the "hot" topics in contemporary metamemory research is the extent to which metamemory is based on people's direct experiences of processing fluency or on people's explicit beliefs about memory. Mendes et al. (2020) address this issue in the context of the effect of word frequency on predictions of future memory performance (judgments of learning, JOLs). Three experiments showed that JOLs made immediately after studying each word were more strongly affected by actual word frequency than by explicit information about word frequency. These findings suggest that the word frequency effect in metamemory relies more heavily on direct experiences than on beliefs. Kuhlmann et al. (2020) examine the contribution of explicit memory beliefs to various metamemory illusions. Across three independent samples, they observed that metamemory illusions about the memorial consequences of different perceptual stimulus characteristics (font type, volume, font size) can be explained by one latent factor. Importantly, however, this factor was unrelated to the belief that high perceptual fluency benefits memory or to the belief that more is better.

Another important topic in contemporary metamemory research focuses on the consequences of memory 
monitoring. Mitton and Fiacconi (2020) investigate this issue in the relatively unexplored domain of metamemory predictions for picture recognition memory. In two experiments, they found that learners spontaneously utilized test experiences to better appreciate their memory abilities, and then applied this knowledge when making subsequent memory predictions for new materials.

Tekin and Roediger (2020) follow-up on recent evidence that metacognitive judgments modify memory rather than merely assess it. Three experiments demonstrated that reactive effects of JOLs generalized to a levels-ofprocessing paradigm and shed new light on the processes responsible for reactive effects of metamemory judgments. Halamish and Undorf (2020) opine that demonstrations of JOL reactivity challenge a fundamental assumption in cognitive-experimental research on metamemory, namely, the assumption that metacognitive monitoring occurs spontaneously and inevitably as cognitive processes take place. They therefore call for addressing novel research questions and for an integration of cognitive-experimental metacognition research with educational-developmental metacognition research.

Finally, Murphy and Castel (2020) introduce the notion of responsible remembering - the strategic allocation of attention toward important information. They propose that responsible remembering can enhance metamemory in both younger and older people. Responsible remembering may improve learning outcomes and prevent that unexpected forgetting produces undesirable outcomes and potentially tragic consequences.

Taken together, the nine papers in this topical issue provide a valuable contribution to the literature on metamemory. The presented new methods will help to clarify unresolved theoretical and empirical issues in metamemory research. The reported findings advance our understanding of yet underexplored aspects of the basis, validity, and consequences of metamemory. Finally, the collection of articles points to links between metamemory and related concepts, suggests how different perspectives on metamemory may be integrated, and identifies gaps in our knowledge about metamemory that deserve attention in future research.

\section{References}

Dobbins, I. G., \& Kantner, J. (2020). The language of recollection in support of recognition memory decisions. Zeitschrift für Psychologie, 228(4), 291-295. https://doi.org/10.1027/2151-2604/ a000426
Flavell, J. H. (1971). First discussant's comments: What is memory development the development of? Human Development, 14(4), 272-278. https://doi.org/10.1159/000271221

Halamish, V., \& Undorf, M. (2020). Do learners spontaneously monitor their memory? The gap between educationaldevelopmental and cognitive-experimental research. Zeitschrift für Psychologie, 228(4), 304-305. https://doi.org/10.1027/ 2151-2604/a000429

Kälin, S., \& Roebers, C. M. (2020). Time-based measures of monitoring in association with executive functions in kindergarten children. Zeitschrift für Psychologie, 228(4), 244-253. https://doi.org/10.1027/2151-2604/a000422

Kelley, T., Serra, M. J., \& Davis, T. (2020). Toward a neurocognitive understanding of the algorithms that underlie metamemory judgments. Zeitschrift für Psychologie, 228(4), 233-243. https://doi.org/10.1027/2151-2604/a000421

Kuhlmann, B. G., Frank, D. J., \& Danner, D. (2020). Louder = larger = clearer: Examining the consistency of metamemory illusions. Zeitschrift für Psychologie, 228(4), 296-300. https://doi.org/ 10.1027/2151-2604/a000427

Mendes, P. S., Luna, K., \& Albuquerque, P. B. (2020). Experience matters: Effects of (in)congruent prompts about word frequency on judgments of learning. Zeitschrift für Psychologie, 228(4), 254-263. https://doi.org/10.1027/2151-2604/a000423

Mitton, E. E., \& Fiacconi, C. M. (2020). Learning from (test) experience: Testing without feedback promotes metacognitive sensitivity to near-perfect recognition memory. Zeitschrift für Psychologie, 228(4), 264-277. https://doi.org/10.1027/21512604/a000424

Murphy, D. H., \& Castel, A. D. (2020). Responsible remembering: How metacognition impacts adaptive selective memory. Zeitschrift für Psychologie, 228(4), 301-303. https://doi.org/ 10.1027/2151-2604/a000428

Nelson, T. O., \& Narens, L. (1990). Metamemory: A theoretical framework and new findings. In G. Bower (Ed.), The psychology of learning and motivation (Vol. 26, pp. 125-173). Academic Press.

Tekin, E., \& Roediger, H. L. (2020). Reactivity of judgments of learning in a levels-of-processing paradigm. Zeitschrift für Psychologie, 228(4), 278-290. https://doi.org/10.1027/21512604/a000425

\section{History}

Published online October 30, 2020

\section{Funding}

Monika Undorf was supported by a Margarete von Wrangell fellowship from the state of Baden-Württemberg.

\section{Monika Undorf}

Department of Psychology

School of Social Sciences

University of Mannheim

A 5, 6, C 203

68159 Mannheim

Germany

undorf@uni-mannheim.de 